\title{
Practice of Extracorporeal Therapies for Septic Acute Kidney Injury Patients in Intensive Care Units in Mainland China
}

\author{
Yu Zhou ${ }^{\text {a }}$ Fachun Zhou $^{\mathrm{b}}$ Xue Wang $^{\mathrm{c}}$ Ping Chang $^{\mathrm{d}}$ Ling Zhang $^{\mathrm{e}}$ \\ Qiang Yao $^{\text {a }}$ Jacques Goldstein ${ }^{f}$ Xinyu Liu ${ }^{a}$ Minmin Wang ${ }^{g}$
}

${ }^{a}$ Medical Affairs, Baxter (China) Investment Co, Ltd, Shanghai, China; ${ }^{b}$ Department of Emergency and Intensive Care Medicine, The First Affiliated Hospital of Chongqing Medical University, Chongqing, China; ' ${ }^{\mathrm{D}}$ Department of Critical Care Medicine, The First Affiliated Hospital of Xi'an Jiaotong University, Xi'an, China; ${ }^{\mathrm{d}}$ Department of Intensive Care Unit, Zhujiang Hospital, Southern Medical University, Guangzhou, China; e Division of Nephrology, West China Hospital of Sichuan University, Chengdu, China; ${ }^{\mathrm{f}}$ Medical Affairs, Baxter International, Braine-l'Alleud, Belgium; g Medical Affairs, Baxter Healthcare (Asia) Pte Ltd, Singapore, Singapore

\section{Keywords}

Septic acute kidney injury · Continuous renal replacement therapy practice $\cdot$ Outcome $\cdot$ Challenge

\begin{abstract}
Background: Continuous renal replacement therapy (CRRT) and other extracorporeal therapies for acute kidney injury (AKI) and other organ dysfunction syndromes in critically ill patients are common in the intensive care unit (ICU). Many studies have focused on clinical practice for managing these conditions. However, there are few studies that describe the utilization of extracorporeal therapies, especially CRRT, in patients with sepsis-associated AKI. Summary: Two hundred ICU physicians were included in a survey from February 28, 2017, to March 20,2017, on the current status of septic AKI and clinical practice in CRRT. According to the responses,
\end{abstract}

$40 \%$ of sepsis patients in the ICU had $\mathrm{AKI}$, and $25 \%$ required extracorporeal therapies. However, $29 \%$ of candidates gave up therapy for medical or nonmedical reasons. Overall survival for sepsis was 60\%; among survivors, $80 \%$ were dialysis free at discharge. CRRT was the most common modality of extracorporeal therapy in the ICU, and $82 \%$ of physicians chose convection as the major clearance mode. The survey showed $30 \%$ of physicians saw the removal of inflammatory mediators as the major objective of extracorporeal therapies; however, only $18.5 \%$ of physicians considered inflammation as a measure to trigger CRRT. The median treatment duration of CRRT in China was 12 h per day for 5 days. Key Messages: There were some similarities and differences in CRRT practice for septic AKI patients in China and globally. The differences reveal some insights into improving the outcomes of these patients.

(C) 2019 The Author(s) Published by S. Karger AG, Basel

\begin{tabular}{ll}
\hline KARGER & $\begin{array}{l}\text { (๑) } 2019 \text { The Author(s) } \\
\text { Published by S. Karger AG, Basel Operger }\end{array}$ \\
E-Mail karger@karger.com & This article is licensed under the Creative Commons Attribution- \\
www.karger.com/bpu & $\begin{array}{l}\text { NonCommercial-NoDerivatives 4.0 International License (CC BY- } \\
\text { NC-ND) (http://www.karger.com/Services/OpenAccessLicense). } \\
\text { Usage and distribution for commercial purposes as well as any dis- } \\
\text { tribution of modified material requires written permission. }\end{array}$
\end{tabular}

Minmin Wang 


\section{Introduction}

Sepsis is the most common cause of death in noncoronary intensive care units (ICUs) [1]. The mortality of ICU patients with severe sepsis and septic shock (definition of Sepsis 1.0 ) is $40.4 \%$, compared with $6 \%$ in patients without sepsis [2]. Meanwhile, growing attention is being paid to acute kidney injury (AKI) because of its increasing incidence and adverse impact on patient outcomes, as well as its health cost burden [3]. Sepsis is the leading cause of AKI; thus, between 45 and 70\% of all AKI is considered to be associated with sepsis, with a high mortality rate of up to $70.2 \%$ among these patients [4]. Although mortality has decreased to some extent in recent years because of advances in technology, it is still high, ranging from 28 to $51.4 \%$ [5-7]. This indicates that our ability to prevent and treat septic AKI is restricted as a result of limited understanding of its pathophysiology [3].

It is widely accepted that hypercytokinemia plays a pivotal role in the pathophysiology of sepsis [8]. Extracorporeal therapies have been proposed as a therapeutic tool against sepsis that functions through the removal of cytokines [8]. However, it remains controversial. Application of extracorporeal therapies in China has been influenced by not only the patient's clinical condition but also the clinician's knowledge and experience.

The aim of the survey was to understand the current perception and clinical practice of extracorporeal therapies, particularlycontinuous renal replacement therapy (CRRT), in septic AKI patients among ICU physicians in China.

\section{Methods}

Description of the Survey and Respondents

The survey was conducted on the Ding Xiang Yuan platform; this is the largest online community for physicians and health-care professionals in China, with more than 5.5 million members [9]. The questionnaire was distributed to $200 \mathrm{ICU}$ physicians between February 28, 2017, and March 20, 2017, and focused on the clinical practice of extracorporeal therapies in sepsis patients.

One of the strict requirements of the survey was the inclusion of only one physician from each department of a particular hospital. The survey data were analyzed based on the assumption that responses from a physician practicing in a specific department of a given hospital would be representative of all the physicians in that department with regard to extracorporeal therapies. In turn, this assumption was predicated upon the observation that most institutions employ therapy protocols that largely standardize local clinical practice.

The Chinese physicians participating in the survey were chosen with the goal of achieving equal distributions with respect to job title (Vice/Chief, Attending, and Resident), hospital type (Class 2 and 3), and city size (Tier 1, 2, and 3). Definition of city size [10]: Tier 1: 4 largest cities in China (Beijing, Shanghai, Guangzhou, and Shenzhen); Tier 2: large provincial capitals and coastal cities, such as Xiamen, Chongqing, Chengdu, and Wuhan; Tier 3: provincial cities of medium size, such as Xuzhou, Handan, and Yantai. Definition of hospital ranking [11]: Class 2: typically found in cities of medium size and have between 100 and 500 beds - in addition to providing health care, they are usually also engaged in medical education and research on a regional basis; Class 3: large institutions (more than 500 beds) that provide both general and specialist care from a large referral base, while also actively supporting medical education and research.

The study was conducted using an online questionnaire, it accessed via email, SMS, and WeChat. Invitations to participate in the study were sent randomly to 2,142 physicians; 488 physicians who were interested in participating were apprised further of the study through an email link. The link provided the opportunity to confirm their agreement to continue with and complete the questionnaire. The respondents were enrolled sequentially in the survey on a "first come, first served" basis, with the primary criteria being the distribution of city size, hospital rank, subspecialty, and job title, as described above.

The responders in the study agreed to the survey responses being analyzed and published. No patient-specific data were provided at any point during the study. Approval was obtained from the independent Ethics Committee of the Shanghai Clinical Research Center (No. 2017-15-01).

\section{Description of the Questionnaire}

The questionnaire included 3 parts: (1) information about the respondent's working facilities and experience in CRRT, as well as the number of new sepsis patients per month in their department; (2) current status of septic AKI and its treatment with extracorporeal therapy; and (3) prescription for CRRT, which included therapy purpose, indication, vascular access, modality, dosage, and anticoagulation methods.

\section{Statistical Analyses}

Data were expressed as mean \pm SD for continuous normally distributed variables, median (interquartile range $[\mathrm{IQR}]$ ) for continuous nonnormally distributed variables, and frequency (percentage) for categorical variables. Differences between groups, such as in city tier or hospital rank, were assessed using the $t$ test for continuous normally distributed variables, the Wilcoxon test for nonnormally distributed variables, and $\chi^{2}$ tests for categorical variables. Statistical analyses were performed using the SPSS 25 software (IBM).

\section{Results}

\section{Characteristics of Survey Respondents and}

CRRT Infrastructure

The distribution of the characteristics of the 200 ICU physicians matched the expected distribution of city size, hospital rank, subspecialty, and job title (Table 1). Most respondents $(51.5 \%)$ were attending physicians, with an average of 5 years' experience in managing extracorpo- 
Table 1. Characteristics of survey respondents $(n=200)$

\begin{tabular}{lc}
\hline Survey period & February 28 - March 20, 2017 \\
\hline City tier, $n(\%)$ & \\
Tier 1 & $67(33.5)$ \\
Tier 2 & $67(33.5)$ \\
Tier 3 & $66(33.0)$ \\
Hospital class, $n$ (\%) & \\
Class 3 & $136(68.0)$ \\
Class 2 & $64(32.0)$ \\
Job title, $n(\%)$ & $56(28.0)$ \\
Vice/Chief & $103(51.5)$ \\
Attending & $41(20.5)$ \\
Resident & $5(3-8)$ \\
Years of CRRT experience, median (IQR) & $8(4-15)$ \\
Number of new sepsis patients per month, median (IQR) & $16(12-22)$ \\
Number of ICU beds, median (IQR) & $2(1-3)$ \\
Number of CRRT equipment, median (IQR) & \\
\hline
\end{tabular}

IQR, interquartile range; CRRT, continuous renal replacement therapy; ICU, intensive care unit.

real therapy. Physicians practicing at 200 hospitals in China participated in the survey, reflecting a broad spectrum of viewpoints.

\section{Snapshot of Sepsis and AKI}

The survey revealed a median of 16 beds, 2 CRRT monitors, and 8 new sepsis patients per month in each ICU (Table 1). Thus, we can estimate a total of 160 sepsis patients per month in the survey.

It is estimated by respondents that $40 \%$ (IQR, 30, 70\%) of sepsis patients progressed to septic shock in the ICU. Pneumonia was the most common infection, accounting for $60 \%$ (IQR, $49,80 \%$ ) of sepsis patients, followed by abdominal and urinary system infections at $20 \%$ (IQR, 10 , $30 \%$ ) and $10 \%$ (IQR, 5, 20\%), respectively. Almost all ICUs measure C-reactive protein (100\%) and procalcitonin $(98 \%)$ levels as a diagnostic indication of sepsis, with less than half of physicians choosing inflammatory mediators (35\%) or endotoxin (34\%) levels. Higher ranking hospitals valued inflammatory mediators more than lower ranking hospitals for sepsis patients ( $42 \%$ in Class 3 vs. $20 \%$ in Class $2, p=0.003$ ).

Approximately $40 \%$ (IQR, 20, 60\%) of sepsis patients also had AKI, while $25 \%$ (IQR, 10, 50\%) of sepsis patients required extracorporeal therapy in the ICU. Among patients who needed extracorporeal therapy, most discontinued this due to "high treatment cost," followed by "old age" and "critical disease condition without hope," according to this survey.

Survey on CRRT Practice for Septic AKI Patients in Mainland China

\section{Treatment Modality for Septic AKI}

CRRT is the most adopted modality for sepsis patients requiring extracorporeal therapy in China (99\%), followed by plasma exchange $(37 \%)$ and intermittent hemodialysis (25\%). Moreover, senior physicians were more inclined to use CRRT (Vice/Chief: 95\%, Attending: $90 \%$, Resident: $80 \%, p=0.008$ ) over other techniques. However, the use of CRRT was not related to city tier (Tier 1: $90 \%$, Tier 2: $95 \%$, Tier 3: $90 \%, p=$ 0.722 ) or hospital rank (Class 2: $96.5 \%$, Class 3: $90 \%$, $p=0.49)$.

\section{Main Purpose and Trigger for CRRT Initiation}

The 2 main purposes of CRRT are renal replacement and the removal of inflammatory mediators in $60 \%$ (IQR, $40,80 \%$ ) and $30 \%$ (IQR, 20, 50\%) of sepsis patients who required extracorporeal therapy, respectively. However, only $18.5 \%$ of physicians considered the degree of inflammation as the most important trigger for CRRT (Fig. 1). While renal replacement was the main objective of CRRT for $60 \%$ of respondents, as mentioned above, renal replacement was the main trigger for CRRT in sepsis patients for $73 \%$ of respondents, which included urine volume and fluid status (42\%), urea nitrogen and creatinine $(29.5 \%)$, and electrolytes (1.5\%). Approximately $35 \%$ of sepsis patients could receive RRT within $6 \mathrm{~h}$ of being diagnosed, and more than $60 \%$ of sepsis patients could receive RRT treatment within $12 \mathrm{~h}$ of being diagnosed. 
Fig. 1. Contradiction between main purpose and trigger for CRRT in sepsis patients. CRRT, continuous renal replacement therapy.

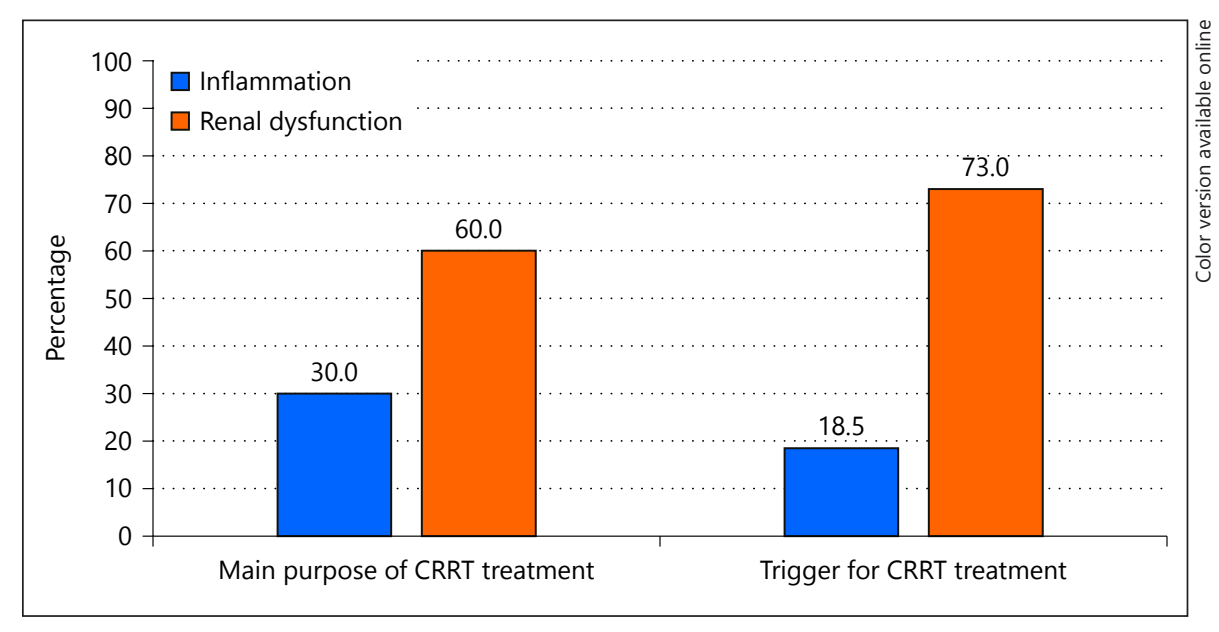

\section{Prescription of CRRT}

The femoral vein was the dominant vascular access site chosen by respondents ( $81 \%$ ), followed by the internal jugular vein (18\%). Of the physicians surveyed, 63 and 19\% chose continuous venovenous hemofiltration and continuous venovenous hemodiafiltration as the modality of CRRT, respectively, which means that convection was chosen by $82 \%$ of respondents. Approximately $74 \%$ of physicians reported prescribing a CRRT dose in the range of $20-30 \mathrm{~mL} / \mathrm{kg} / \mathrm{h}$, while $20 \%$ preferred $30-45 \mathrm{~mL} / \mathrm{kg} / \mathrm{h}$, with $1 \%$ choosing a very high prescribing dose of more than $45 \mathrm{~mL} / \mathrm{kg} / \mathrm{h}$. The CRRT treatment duration per patient was $5(3,7)$ days and $12(8,24)$ hours per day. However, physicians who preferred a high dose of more than $30 \mathrm{~mL} / \mathrm{kg} / \mathrm{h}$ went for a shorter treatment duration per day $(8 \mathrm{~h})$ than those who preferred prescribing a dose of $<30 \mathrm{~mL} / \mathrm{kg} / \mathrm{h}$ (12 h). Sepsis patients for whom renal replacement was the aim received a longer treatment duration than those for whom removal of inflammatory mediators was the aim (7 vs. 5 days, $p<0.0001)$. Unfractionated heparin (35\%), lowmolecular-weight heparin (34\%), and citrate (29\%) were most commonly used during CRRT for anticoagulation.

\section{Outcomes of Septic AKI}

Median survival of septic AKI patients was $60 \%$ (IQR, $40,80 \%$ ); $80 \%$ (IQR, 70, 95\%) of survivors had full or partial renal recovery. A higher hospital rank was linked to greater survival (65\% in Class 3 hospitals vs. 50\% in Class 2 hospitals). However, there were no differences in renal recovery among different city tiers and hospital ranks. Initiation time of RRT was considered the most important factor for both mortality and renal recovery, regardless of city tier and hospital rank, as well as job title, CRRT experience, and sepsis management experience.

\section{Challenges and Actions to Improve Outcomes}

The ability of the patient to pay was most widely considered as the main challenge in CRRT for septic AKI patients. Apart from this, absence of a therapeutic effect index and definite initiation time were 2 other significant challenges considered by more than half of the participating physicians - 58 and 52\%, respectively - regardless of city tier, hospital rank, job title, CRRT experience, and sepsis management experience. Half of the participant hospitals have no outpatient follow-ups after patients recover and are discharged from the ICU/hospital.

In total, $90 \%$ of ICU physicians thought that standardized treatment was the most important measure to improve sepsis outcomes. Qualification assessment was considered as the second most important measure, as selected by $69 \%$ of participant physicians. Approximately $34 \%$ of physicians believed that improved filter performance via new technologies could further improve outcomes.

\section{Discussion and Conclusion}

While studies assessing RRT practices with AKI in China have been previously published, this is the first survey focused on septic AKI population and it's extracorporeal therapy, as the surveyed physicians represented a broad spectrum with regard to seniority, hospital ranking, and city size, we believe that the survey is generally representative of extracorporeal therapy practices in Chinese ICUs as a whole.

In the survey, the epidemiology of septic AKI was similar to that in China and in other countries: $40 \%$ of sepsis patients had AKI, a similar proportion to those in previ- 
ous multicenter studies conducted in ICUs (range 39.4$53.2 \%)[5,12-14]$. Infection sites that caused sepsis were also similar to those observed in a previous study [6], this is the only single-center study, which indicated that $23.3 \%$ of septic AKI patients required extracorporeal therapy, our survey is the first on a much larger scale to estimate this proportion at $25 \%$ in Chinese ICUs. Overall survival of septic AKI patients in ICUs was $60 \%$ in this survey, which is similar to the previous study in China reported by Wang et al. [5]. In addition, among surviving patients, $80 \%$ were independent of dialysis at discharge, a proportion that also closely resembles that in a previous study in China [15]. Furthermore, CRRT modality [16], the main prescription dose of CRRT [17], and overall treatment duration $[18,19]$ were all consistent with worldwide CRRT practices.

Compared with sustained low-efficiency dialysis, which is seldom used, CRRT was dominantly accepted by ICU physicians for septic AKI patients; this is mainly driven by patients in the ICU setting, in which intensivists independently manage their therapy. Other extracorporeal therapy techniques were also found to be common for sepsis patients, such as plasma exchange and hemoperfusion, similar to the recent international survey by Ronco et al. [20]; these techniques have been increasingly used in recent years compared with previous studies [21]. This trend is present even if the current literature does not actually recommend the application of extracorporeal therapy in the clinical practice of sepsis management. Possibly, this is a field in which increased education should be delivered [21].

However, there are other aspects of CRRT practice in China that lag behind the developed world to some extent. Thus, there may be some effective strategies to improve the outcomes of septic AKI patients according to this survey. First, an improved effective dose delivered on a daily basis could be attained through extending the treatment duration per day, as it always falls below international standards [17] because of relatively short treatment durations (only $12 \mathrm{~h}$ ) per day, particularly for high prescription doses $(>30 \mathrm{~mL} / \mathrm{kg} / \mathrm{h})$, which last for only $8 \mathrm{~h}$ per day, according to both previous [22] and current surveys. Second, heparin-based anticoagulation was the dominant anticoagulation method; the utilization of regional citrate anticoagulation was only $29 \%$ in China, much lower than in the developed world [23]; this may increase the bleeding risk as citrate has a more favorable safety profile than heparin, as recommended by Kidney Disease Improving Global Outcomes [17]. The reason for the low utilization of citrate may be a lack of com-

Survey on CRRT Practice for Septic AKI Patients in Mainland China mercialized citrate fluid, with the only choice for Chinese physicians being compounded fluid, which not only increases the risk of infection but also adds work load to the CRRT operator. Third, there was already a degree of awareness that CRRT could remove inflammatory mediators in septic AKI patients, and 30\% of CRRT users saw it as the major objective of CRRT treatment; however, only $19 \%$ of physicians perceived inflammation as a trigger for CRRT. The gap between the main purpose and trigger index is probably a result of physicians still being uncertain about the effect of removing inflammatory mediators, as well as not being sure of how to choose the right patients and initiation time based on the inflammatory mediator index. More effort should be made to educate physicians on how to identify the right patients. Fourth, ICU physicians in China prefer the femoral vein to the internal jugular vein as the dominant vascular access, in contrast to the Kidney Disease Improving Global Outcomes guidelines [17]. This may have arisen from some local Chinese guidelines from several years ago [24], which suggested access via the femoral vein as the first choice as it is easier to access, similar safety profile, and has the same infection rate as the internal jugular vein. Fifth, this survey showed that half of the participating hospitals have no nephrology followup for septic AKI patients, which will certainly have a negative impact on prognosis as physicians cannot draw lessons from the past. Collaboration with nephrology departments may be an operational solution for ICU physicians to collect the follow-up information of septic AKI patients. Finally, a high proportion of septic AKI patients judged to be RRT candidates gave up extracorporeal therapy because of nonmedical reasons, such as the high treatment cost. Insurance policies vary considerably across the country at present [25], resulting in the need for some patients and their families to make very difficult decisions about potentially life-saving medical technologies. Therefore, it is important to improve the insurance situation to reduce the barriers to CRRT utilization.

Although our study provided important insights into extracorporeal therapy, especially CRRT practice for septic AKI in China, it has some limitations. The data generated from the survey were relatively qualitative in nature and may not reflect Chinese clinical practice in all respects.

This survey demonstrated similarities in the current status of septic AKI and awareness of cytokine removal among ICU physicians in China and other countries. However, as a result of medical and nonmedical resource 
limitations, differences in CRRT practice still exist in China compared with the developed world; therefore, there is a long way to go to further improve the prognosis for septic AKI patients, and increased education should be delivered simultaneously.

\section{Acknowledgment}

None.

\section{Statement of Ethics}

The authors have no ethical conflicts to disclose.

\section{Disclosure Statement}

M.W., J.G., X.L., Q.Y. and Y.Z. are Baxter employees and hold Baxter stocks. All other authors have no conflicts of interest to declare.

\section{Funding Sources}

NA.

\section{Author Contributions}

Y.Q., W.M., and Z.Y. designed and implemented the survey and contributed to manuscript writing and editing. All authors discussed and approved the final manuscript.

\section{References}

1 Liu V, Escobar GJ, Greene JD, Soule J, Whippy A, Angus DC, et al. Hospital deaths in patients with sepsis from 2 independent cohorts. JAMA. 2014 Jul;312(1):90-2.

2 SepNet Critical Care Trials Group. Incidence of severe sepsis and septic shock in German intensive care units: the prospective, multicentre INSEP study. Intensive Care Med. 2016 Dec;42(12):1980-9.

3 Bellomo R, Kellum JA, Ronco C, Wald R, Martensson J, Maiden M, et al. Acute kidney injury in sepsis. Intensive Care Med. 2017 Jun;43(6):816-28.

4 Bagshaw SM, Uchino S, Bellomo R, Morimatsu H, Morgera S, Schetz M, et al.; Beginning and Ending Supportive Therapy for the Kidney (BEST Kidney) Investigators. Septic acute kidney injury in critically ill patients: clinical characteristics and outcomes. Clin J Am Soc Nephrol. 2007 May;2(3):431-9.

5 Wang X, Jiang L, Wen Y, Wang MP, Li W, Li $\mathrm{ZQ}$, et al. Risk factors for mortality in patients with septic acute kidney injury in intensive care units in Beijing, China: a multicenter prospective observational study. BioMed Res Int. 2014;2014:172620.

6 Shum HP, Kong HH, Chan KC, Yan WW, Chan TM. Septic acute kidney injury in critically ill patients - a single-center study on its incidence, clinical characteristics, and outcome predictors. Ren Fail. 2016 Jun;38(5): 706-16.

7 Medeiros P, Nga HS, Menezes P, Bridi R, Balbi A, Ponce D. Acute kidney injury in septic patients admitted to emergency clinical room: risk factors and outcome. Clin Exp Nephrol. 2015 Oct;19(5):859-66.

8 Hirasawa $\mathrm{H}$, Oda S, Nakamura M, Watanabe E, Shiga H, Matsuda K. Continuous hemodi- afiltration with a cytokine-adsorbing hemofilter for sepsis. Blood Purif. 2012;34(2):164-70.

9 DXY [Internet]. Company profile [cited 2019 Mar 5]. Available from: http://www.dxy.cn/ pages/about.html

10 Chinese Government Network [Internet]. Standard of city scale: the state council of the People's Republic of China. 2014 [cited 2019 Mar 5]. Available from: http://www.gov.cn/ zhengce/content/2014-11/20/content_9225. htm

11 Bagshaw SM, George C, Bellomo R; ANZICS Database Management Committee. Early acute kidney injury and sepsis: a multicentre evaluation. Crit Care. 2008;12(2):R47.

12 Oppert M, Engel C, Brunkhorst FM, Bogatsch H, Reinhart K, Frei U, et al.; German Competence Network Sepsis (Sepnet). Acute renal failure in patients with severe sepsis and septic shock-a significant independent risk factor for mortality: results from the German Prevalence Study. Nephrol Dial Transplant. 2008 Mar;23(3):904-9.

13 Poukkanen M, Vaara ST, Pettilä V, Kaukonen $\mathrm{KM}$, Korhonen AM, Hovilehto S, et al.; FINNAKI study group. Acute kidney injury in patients with severe sepsis in Finnish Intensive Care Units. Acta Anaesthesiol Scand. 2013 Aug;57(7):863-72.

14 Tian H, Sun T, Hao D, Wang T, Li Z, Han S, et al. The optimal timing of continuous renal replacement therapy for patients with sepsisinduced acute kidney injury. Int Urol Nephrol. 2014 Oct;46(10):2009-14.

15 Clark WR, Ronco C. Continuous renal replacement techniques. Contrib Nephrol. 2004;144:264-77.

16 Kidney Disease: Improving Global Outcomes (KDIGO) Acute Kidney Injury Work Group.
KDIGO clinical practice guideline for acute kidney injury. Kidney Int Suppl. 2012;2(1): $1-138$.

17 Palevsky PM, Zhang JH, O'Connor TZ, Chertow GM, Crowley ST, Choudhury D, et al.; VA/NIH Acute Renal Failure Trial Network. Intensity of renal support in critically ill patients with acute kidney injury. $\mathrm{N}$ Engl J Med. 2008 Jul;359(1):7-20.

18 Claure-Del Granado R, Mehta RL. Fluid overload in the ICU: evaluation and management. BMC Nephrol. 2016 Aug;17(1):109.

19 Digvijay K, Neri M, Fan W, Ricci Z, Ronco C. International survey on the management of acute kidney injury and continuous renal replacement therapies: Year 2018. Blood Purif. 2019;47(1-3):113-9.

20 Basso F, Ricci Z, Cruz D, Ronco C. International survey on the management of acute kidney injury in critically ill patients: year 2007. Blood Purif. 2010;30(3):214-20.

21 Clark WR, Ding X, Qiu H, Ni Z, Chang P, Fu $P$, et al. Renal replacement therapy practices for patients with acute kidney injury in China. PLoS One. 2017 Jul;12(7):e0178509.

22 Oudemans-van Straaten HM. Citrate for continuous renal replacement therapy: safer, better and cheaper. Crit Care. 2014 Dec;18(6): 661

23 Chines Society of Critical Care Medicine [Internet]. Guideline of blood purification in ICU. 2010 [cited 2019 Mar 5]. Available from: http://www.csccm.org/cn/news.asp?id=121. html

24 Zhu H, Liu L, Song A, et al. Studying on the fairness of health insurance system under the new normal of universal health insurance. Chinese Health Serv Manage. 2016;33(7): $512-4$. 